Check for updates

Cite this: RSC Adv., 2018, 8, 30912

\title{
High energetic polymeric nitrogen sheet confined in a graphene matrix
}

\author{
Shifeng Niu, ${ }^{a}$ Shijie Liu, (D) ab Bo Liu, ${ }^{a}$ Xuhan Shi, ${ }^{a}$ Shuang Liu, ${ }^{a}$ Ran Liu, ${ }^{a}$ \\ Mingguang Yao, ${ }^{a}$ Tian Cui (D) and Bingbing Liu (D) *a
}

Polymeric nitrogen, as a potential high-energy-density material (HEDM), has many applications, such as in energy storage systems, explosives and propellants. Nowadays it is very urgent to find a suitable method to stabilize polymeric nitrogen at ambient conditions. Herein, we present a new hybrid structure where polymeric nitrogen sheets are sandwiched between graphene sheets in the form of a three-dimensional crystal. According to ab initio molecular dynamics (AIMD) calculations and phonon spectrum calculations, it is demonstrated that polymeric nitrogen sheets are stable at ambient pressure and temperature. The hybrid material has a higher nitrogen content (the weight ratio of nitrogen is up to $53.84 \%$ ), and the corresponding energy density is $5.2 \mathrm{~kJ} \mathrm{~g}^{-1}$. The hybrid material (A7@graphene system) has a satisfactory energy density, detonation velocity and detonation pressure. Importantly, the hybrid material can be preserved up to $450 \mathrm{~K}$, and above this temperature, the polymeric nitrogen sheets break up into polymeric nitrogen chains or nitrogen gases and release tremendous energy. Further calculations reveal that small charge transfer between the polymeric nitrogen sheets and graphene sheets creates a weak electrostatic attraction compared with other hybrid materials, which is just good for the stabilization of the polymeric nitrogen sheets at ambient conditions, and favors energy release in a gentle way. The proposed confinement hybrid material which has a high energy density and a gentle energy release temperature, provides a highly promising method for the capture and application of polymeric nitrogen in a controllable way.

Received 22nd April 2018 Accepted 28th July 2018

DOI: $10.1039 / c 8 r a 03453 b$

rsc.li/rsc-advances

\section{Introduction}

Due to a uniquely large amount of energy difference between the single $\mathrm{N}-\mathrm{N}$ bond $\left(160 \mathrm{~kJ} \mathrm{~mol}^{-1}\right)$ or double $\mathrm{N}=\mathrm{N}$ bond (418 $\mathrm{kJ} \mathrm{mol}^{-1}$ ) and triple $\mathrm{N} \equiv \mathrm{N}$ bond $\left(954 \mathrm{~kJ} \mathrm{~mol}^{-1}\right.$ ), polymeric nitrogen, ${ }^{1-25}$ which contains single $\mathrm{N}-\mathrm{N}$ and double $\mathrm{N}=\mathrm{N}$ bonds, is causing continuous attention as a potential HEDM for energy storage systems, explosives and propellants. The energy density of polymeric nitrogen is estimated to be at least three times higher than the most powerful energetic materials known today, ${ }^{26}$ such as trinitrotoluene (TNT), cyclotetramethylenetetranitramine (HMX), and so on. Moreover, nitrogen gas, which is the product of polymeric nitrogen decomposition, is friendly to the environment. Owing to the excellent physical and chemical properties of polymeric nitrogen given above, over the past decades, many structures of polymeric nitrogen have been predicted in theory, such as chainlike structures, layered structures, and network structures. The no-molecular nitrogen phase under high pressure

${ }^{a}$ State Key Laboratory of Superhard Materials, Jilin University, Changchun 130012, P. R. China. E-mail: liubb@jlu.edu.cn; Tel: +86-431-85168256

${ }^{b}$ School of Physics and Engineering, Henan Key Laboratory of Photoelectric Energy Storage Materials and Applications, Henan University of Science and Technology, Luoyang 471003, China was first proposed by McMahan et $a .^{25}$ in theory. A few of the polymeric nitrogen structures have been demonstrated at high pressure and temperature conditions in experiment. Firstly, the so-called single-bond polymeric nitrogen $(\mathrm{CG})^{27,28}$ was reported at high pressure (110 GPa) and temperature $(2000 \mathrm{~K})$. Subsequently, a layered polymeric nitrogen form (LP-N) ${ }^{29,30}$ has also been proved in experiment and successfully synthesized at a higher pressure and temperature conditions $(150 \mathrm{GPa}, 3000 \mathrm{~K})$ in 2014 . Nitrides have also made great progress in numerous experimental studies, for example, $\left(\mathrm{N}_{5}\right)_{6}\left(\mathrm{H}_{3} \mathrm{O}\right)_{3} \cdot\left(\mathrm{NH}_{4}\right)_{4} \mathrm{Cl},{ }^{31} \quad$ [2,2'-bi (1,3,4-oxadiazole)-5, $5^{\prime}$-dinitramide], ${ }^{32} \mathrm{Na}_{20} \mathrm{~N}_{60}$ and $\mathrm{Na}_{24} \mathrm{~N}_{60}$ nanocages. ${ }^{33}$ In addition, searching for an effective method to stabilize polymeric nitrogen to ambient conditions also becomes a hot topic, and it is important for the practical application of polymeric nitrogen.

Recently, much attention has been attracted to hybrid materials ${ }^{34-38}$ which polymeric nitrogen chains are confined in one or two-dimensional nano-material in theory and these polymeric nitrogen chains become stable at ambient conditions. By AIMD simulations and the calculated electronic structures, columbic interaction which stems from the charge transfer between the polymeric nitrogen chains and the hosting materials is thought to be the stable mechanism of these hybrid 
materials at ambient conditions. ${ }^{39-42}$ In particular, we also found that the polymeric nitrogen chains encapsulated in boron nitrogen nanotube (BNNT) ${ }^{43}$ are stable at ambient conditions with the same physical mechanism, and the polymeric nitrogen chains dissociate into nitrogen gases and release tremendous energy at above $1400 \mathrm{~K}$. In experiment, we found that the non-gaseous nitrogen is able to obtain by encapsulated in the channels of AFI zeolite at ambient pressure and temperature. ${ }^{44}$ The results of these studies demonstrate that nano-confinement is a promising method to obtain energetic polymeric nitrogen at ambient conditions. ${ }^{43,44}$ However, previous studies are mainly about one or two-dimensional hybrid systems, three-dimensional hybrid system still have not been studied systematically so far. Therefore it is necessary to study whether three-dimensional hybrid system has better properties and the same physics.

A7 polymeric nitrogen is proposed by McMahan et al. ${ }^{25}$ and crystallizes in a rhombohedral structure with a space group of $R \overline{3} m$ and cell parameters of $a=2.401(\AA), b=2.401(\AA), c=7.822$ $(\AA) .{ }^{25,28}$ These nitrogen atoms form a layered structure in the direction of the $c$-axis with polymeric nitrogen sheets. Compared to the previously reported hybrid materials in which polymeric nitrogen chains are encapsulated in one or twodimensional nano-materials, the nitrogen content of the hybrid material will greatly increased by encapsulating polymeric nitrogen sheets into nano-materials, which is very important for the promotion of energy density.

In this work, we propose a new hybrid material-an A7@graphene system in which polymeric nitrogen sheets are sandwiched between graphene sheets in the form of a threedimensional crystal. Phonon spectrum calculation and AIMD simulations confirm that the polymeric nitrogen sheets are stable at ambient conditions. The stable mechanism is revealed by electronic structure analysis. More importantly, the hybrid material has a higher nitrogen content (the weight ratio of nitrogen is up to $53.84 \%$ ), and the corresponding energy density is $5.2 \mathrm{~kJ} \mathrm{~g}^{-1}$. The stability range of the hybrid system is further studied, and we find that the hybrid material can be preserved up to $450 \mathrm{~K}$, and above this temperature, the polymeric nitrogen sheets break up into polymeric nitrogen chains or nitrogen gases and release tremendous energy. Thus we propose a new hybrid material which offers a path for accommodating more polymeric nitrogen in nano-material and extending the application of polymeric nitrogen in a controllable way.

\section{Theoretical calculations}

Our calculations performed in this paper are within the firstprinciples plane-wave pseudopotential density functional theory (DFT) as implemented in the VASP code. ${ }^{45}$ The projected augmented wave (PAW) method is employed with the PAW potentials taken from the VASP library where $2 \mathrm{~s}^{2} 2 \mathrm{p}^{2}$ and $2 \mathrm{~s}^{2} 2 \mathrm{p}^{3}$ are treated as the valence electrons of $\mathrm{C}$ and $\mathrm{N}$ atoms, respectively. The generalized gradient approximation (GGA) PerdewBurke-Ernzerhof (PBE) is used to describe the exchangecorrelation interactions. ${ }^{46-48} \mathrm{~A}$ unit cell containing one graphene sheet and polymeric nitrogen sheet, with two carbon atoms and two nitrogen atoms respectively, is constructed with a reasonable small mismatch. In order to study the electronic structures, we performed atomic relaxation of A7@graphene structure system. The structure is completely relaxed both with respect to atomic positions and unit cell size. A plane-wave cutoff energy $(520 \mathrm{eV})$ for the expansion of the wave function into plane waves ${ }^{49}$ and a proper Monkhorst-Pack $k$-meshes ( $k$-points density $0.03 \AA^{-1}$ ) sampled in the Brillouin zone are used to ensure the maximum atomic force in the system is less than $0.005 \mathrm{eV}$ per atom and the energy difference of two iterations is less than $10^{-6} \mathrm{eV}$ per atom. The vdWs interaction is considered by using the optB86b-vdW functional in calculation. ${ }^{50,51}$ In addition, to test the effectiveness of our method, we performed atomic relaxation of A7 polymeric nitrogen at $100 \mathrm{GPa}$, the N-N bond length is calculated to be $1.41 \AA$, which is in good agreement with previously reported theoretical value $(1.40 \AA) . .^{28,52,53}$ AIMD simulations are performed in constant volume and constant temperature (NVT) ${ }^{54,55}$ for 2 ps time period with the time step of $1 \mathrm{fs}$. During the simulations, we set the step $50 \mathrm{~K}$ or $100 \mathrm{~K}$ in the range of $300-5000 \mathrm{~K}$ by using Nose thermostat. The calculation of net charge is based on the Bader analysis for unbiasedly evaluating the actual charge transfer ${ }^{56}$ The phonon calculation is carried out by using a supercell (finitedisplacement) approach as implemented in the PHONOPY program. ${ }^{57}$ We calculated the detonation velocity and detonation pressure by using the Kamlet-Jacobs simi-empirical equation.$^{58}$ The crystal structure graphics are produced by using the VESTA software.

\section{Results and discussion}

\subsection{Crystal structures}

The optimized atomic structures corresponding to the unit cell and supercell $(4 \times 4 \times 2)$ of A7@graphene system are shown in Fig. 1(a) and (b), respectively. From these two panels, we know that the polymeric nitrogen sheets settles in the middle of graphene sheets and the relaxed distance between the polymeric nitrogen sheets and graphene sheets is $3.905 \AA$. The N-N bond length of the A7 polymeric nitrogen sheets is $1.58 \AA$ (a)

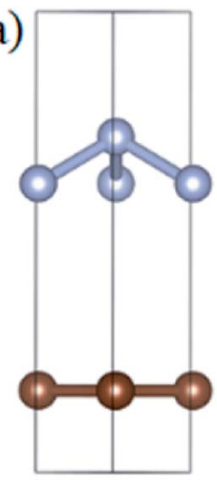

(b)

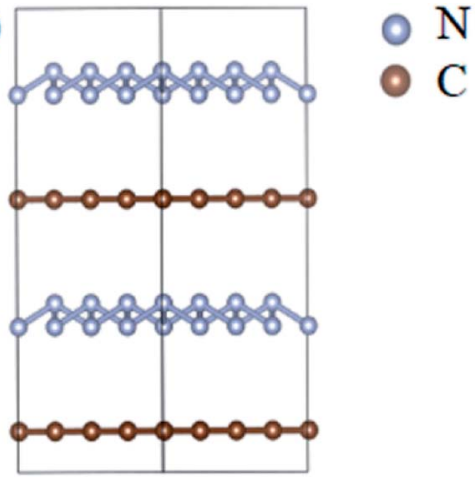

Fig. 1 The side view (left) for (a) the unit cell and (b) supercell $(4 \times 4 \times$ 2) of the A7@graphene crystal structure. The silver-gray and brown spheres are nitrogen atoms and carbon atoms, respectively. 
(calculated at $0 \mathrm{GPa}$ ), which has a slight change compared to the previously reported theoretical value $(1.59 \AA)^{25}$ due to the nanoconfinement effect. The supercell is further used for AIMD simulation calculations.

\subsection{Structural stability analyses}

To study the stability of A7@graphene system, two aspect analyses of the molecular dynamic (MD) stability and dynamic stability are carried out respectively. The evolution of bond lengths as a function of time at $300 \mathrm{~K}$ is given in Fig. 2, which show that the $\mathrm{N}-\mathrm{N}$ bond lengths of A7 polymeric nitrogen sheets have a slightly fluctuation but keep constant in the simulation calculation, which demonstrates that the A7@graphene system is stable at ambient pressure and temperature. Significantly, the phonon calculation is carried out at ambient pressure. The phonon spectrum is shown in Fig. 3. No imaginary phonon frequency is found in the whole Brillouin zone at ambient pressure, which means that the hybrid material is dynamically stable under normal pressure. In addition, the

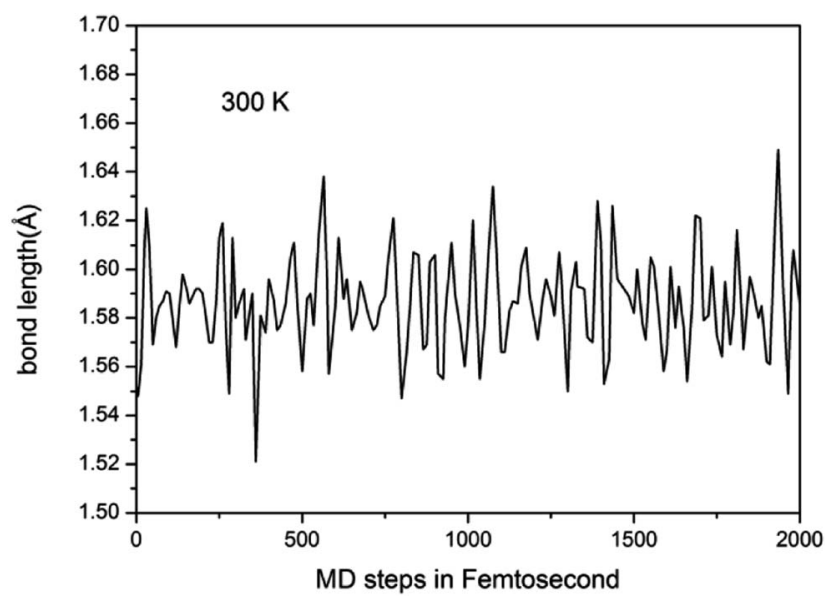

Fig. 2 Lengths of $\mathrm{N}-\mathrm{N}$ bond as a function of time at $300 \mathrm{~K}$ in our molecular dynamic simulations.

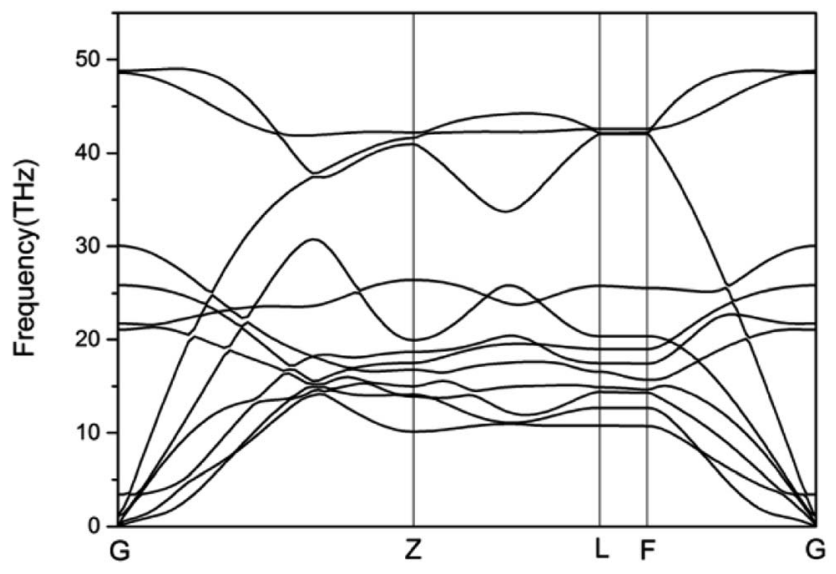

Fig. 3 Phonon-dispersion curves for the A7@graphene crystal structure at ambient pressure.
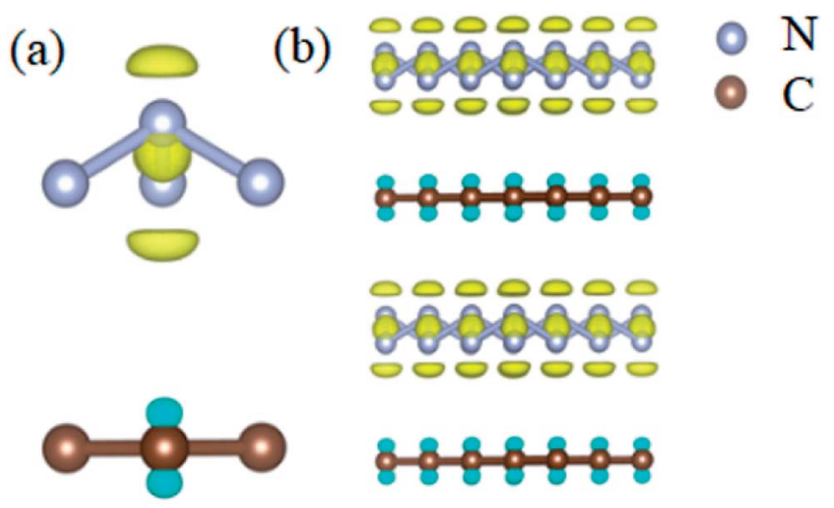

Fig. 4 The isosurface of electron density difference for (a) the unit cell and (b) supercell $(4 \times 4 \times 2)$ of the A7 agraphene crystal structure. Blue and yellow denote the effective positive and negative charge respectively, while the panel is an isosurface of $\pm 2.3 \times 10^{-4}\left(\mathrm{e} \AA^{-3}\right)$. The silver-gray and brown spheres are nitrogen atoms and carbon atoms, respectively.

zero-temperature atomic relaxation also verifies the stability of A7@graphene at ambient pressure.

In order to clarify the physical mechanism of the A7@graphene system stability at ambient conditions, we study the electronic properties of the hybrid material. First, the charge redistribution of the hybrid material is investigated by subtracting electron of the polymeric nitrogen sheets and graphene sheets from the density of A7@graphene system to show the electron distributes due to the charge transfer between polymeric nitrogen sheets and graphene sheets, which are shown in Fig. 4(a) and (b) for the unit cell and supercell $(4 \times 4 \times 2)$ respectively. From the results we know that the electron density is increased around polymeric nitrogen sheets while decreased near the graphene sheets. Therefore, a certain amount of electrons are transferred from graphene sheets to polymeric nitrogen sheets. Furthermore, by using the Bader charge transfer analysis, it is revealed that there are about 0.0049 electrons transferred to polymeric nitrogen sheets $(\mathrm{e} / \mathrm{N})$, which is consistent with the former charge redistribution calculations. The cumulation of negative charges on the nitrogen sheets and positive charges on the graphene sheets give rise to the coulomb interaction between polymeric nitrogen sheets and graphene sheets, which stabilizes the hybrid system at ambient conditions. Similar stabilization mechanism is reported in the case of polymeric nitrogen chains encapsulated in carbon nanotubes $(\mathrm{CNT}),{ }^{34}$ silicon carbide nanotubes (SiCNT), ${ }^{37}$ and BNNT. ${ }^{43}$

We further study the band structures of A7@graphene system (Fig. 5(a)). To highlight the effects of the interaction between the polymeric nitrogen sheet and graphene sheet, we use the same atomic coordinates as in the relaxed A7@graphene system calculating the band structures of the free polymeric nitrogen sheet and empty graphene sheet, which are shown in Fig. 5(b) and (c) for comparison. Based on the comparison of the calculated band structures, we find that the energy bands of the A7@graphene system are basically the superposition of the energy bands of polymeric nitrogen sheet and graphene sheet, i.e. the lowest three conduction bands of A7@graphene system are from the polymeric nitrogen sheet 
(a)

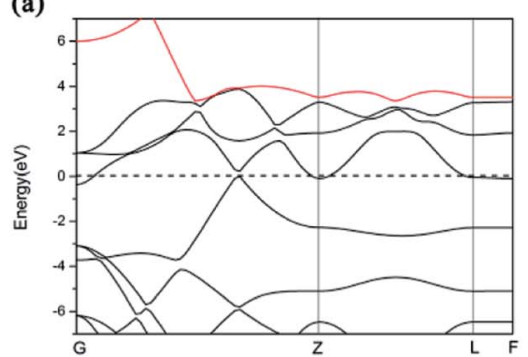

(b)

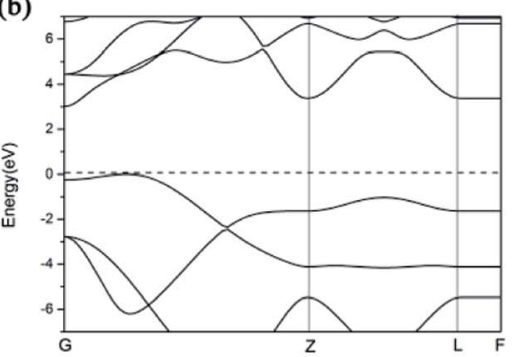

(c)

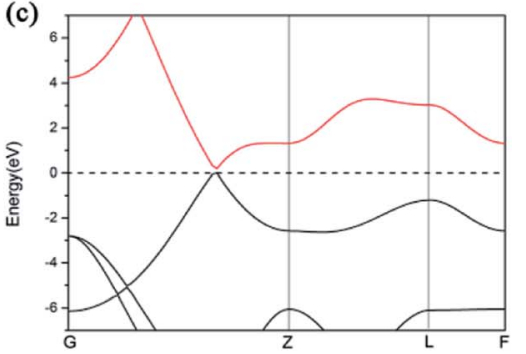

Fig. 5 Band structures for (a) the A7@graphene system, (b) the free polymeric nitrogen sheet, and (c) the empty graphene sheet.

and the top valence band is from graphene sheet, which shows that no hybridization takes place between these corresponding occupied orbits of the polymeric nitrogen sheet and graphene sheet. Nevertheless, the energy bands of the polymeric nitrogen sheet are rigidly lowered, and graphene sheet are rigidly shifted upward due to the interaction between the guest and host. In addition, we also find that the lowest conduction band of graphene sheet in the A7@graphene system is changed slightly compared to the corresponding energy band of the isolated graphene sheet duo to the charge transfer between polymeric nitrogen sheets and graphene sheets.

Furthermore, we find that there is a weak orbital hybridization between the conduction bands of polymeric nitrogen sheets and graphene sheets. The PDOS for the A7@graphene system is shown in Fig. 6(a) and the PDOS for the free polymeric nitrogen sheets and empty graphene sheets are also shown in Fig. 6(b) for comparison. By contrast, we find that the week orbital hybridization happens at $3.85 \mathrm{eV}$ above the Fermi level (Fig. 6(a)). To ensure the specific hybrid orbits, the banddecomposed charge-density analysis of A7@graphene system is carried out too, which is exhibited in Fig. 6(c). From the results, we know that the week orbital hybridization mainly originates from the $\mathrm{N} \_2 p, \mathrm{~N} \_2$ s orbital and $\mathrm{C} \_2 \mathrm{p}$ orbital due to the interaction between the polymeric nitrogen sheets and graphene sheets.
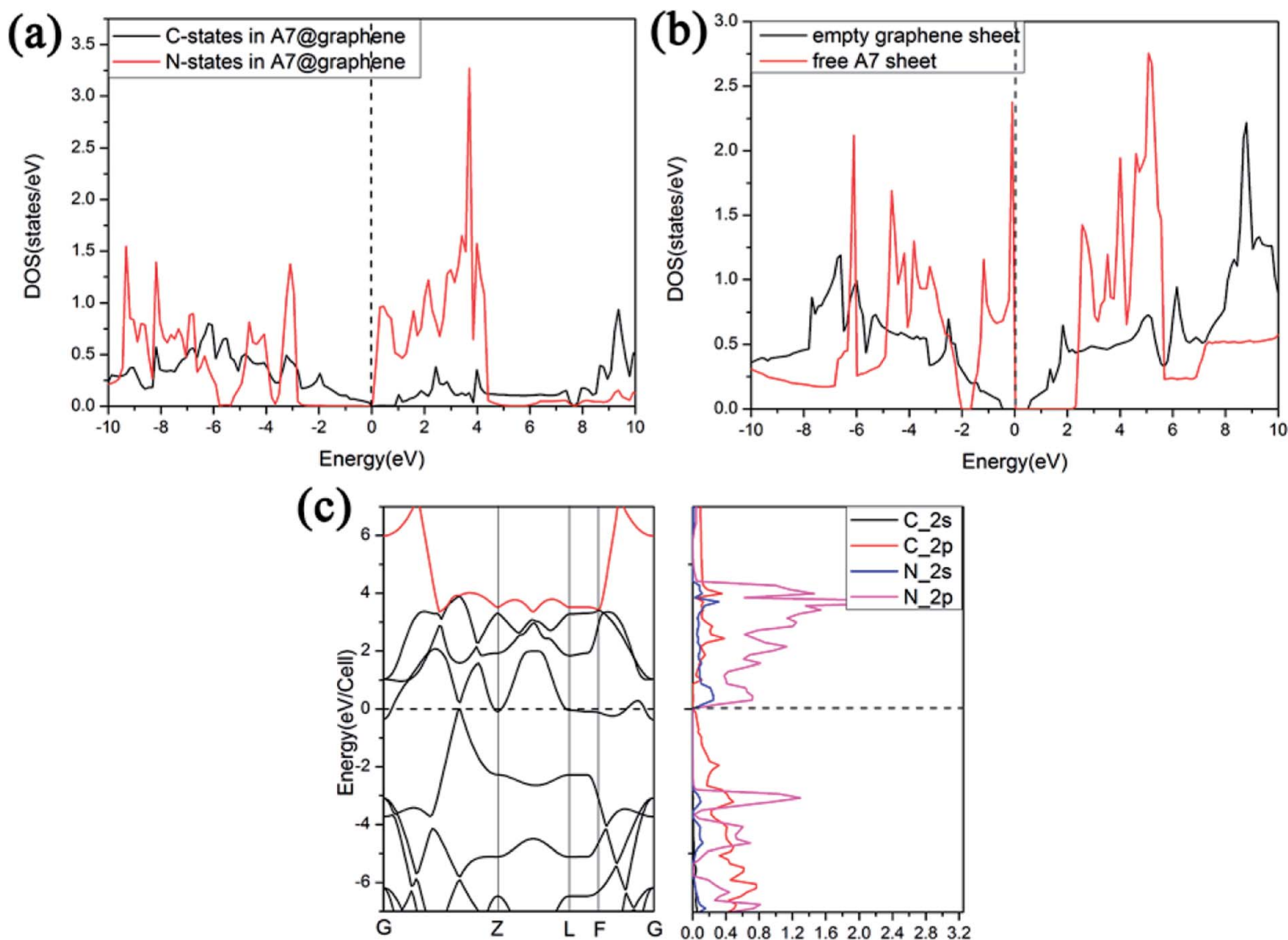

Fig. 6 Projected density of states (PDOS) for (a) the A7@graphene system, and (b) free polymeric nitrogen sheet and empty graphene sheet. (c) The band-decomposed charge-density of the A7@graphene system. A $0.05 \mathrm{eV}$ broadening is used. 


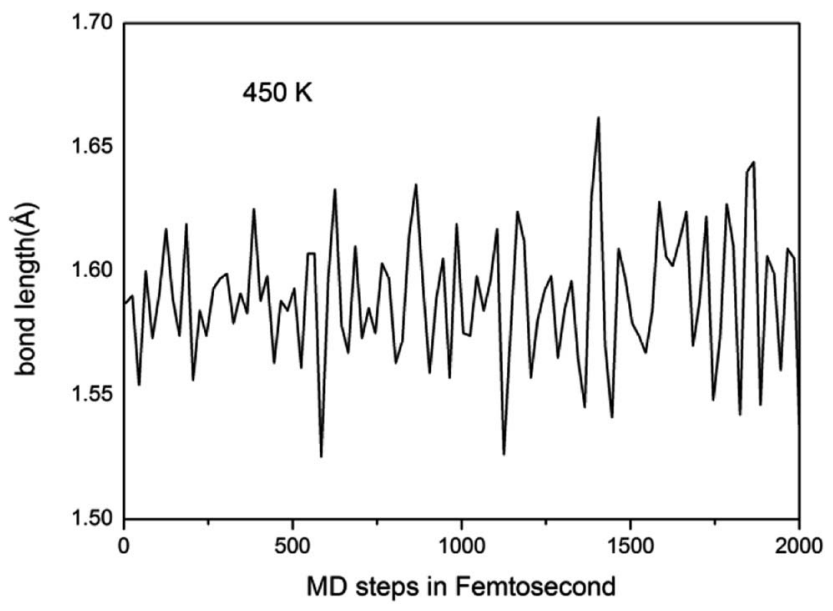

Fig. 7 Lengths of $\mathrm{N}-\mathrm{N}$ bond as a function of time at $450 \mathrm{~K}$ in our molecular dynamic simulations.

\subsection{AIMD simulations analysis}

We further study the stable temperature range of the A7@graphene system by AIMD simulations in the temperature range from $300 \mathrm{~K}$ to $5000 \mathrm{~K}$ for $2 \mathrm{ps}$ time period with the step $50 \mathrm{~K}$ or $100 \mathrm{~K}$. The studies show that the bond lengths of polymeric nitrogen sheets have a slight fluctuation but keep constant in the range of temperature $(300-450 \mathrm{~K})$, and there is no chemical bonding between the polymeric nitrogen sheets and graphene sheets and the dissociation of polymeric nitrogen sheets, which means that the polymeric nitrogen sheets are stable in the range of temperature $(<450 \mathrm{~K})$. The evolution of bond lengths as a function of time at $450 \mathrm{~K}$ is given in Fig. 7. When the temperature is above $450 \mathrm{~K}$, the polymeric nitrogen sheets begin to break up into polymeric nitrogen chains and nitrogen gases firstly. When the temperature is higher $(1800 \mathrm{~K})$, the polymeric nitrogen sheets are completely dissolved into nitrogen gases and release tremendous energy, as shown in Fig. 8, it is confirmed that the polymeric nitrogen sheets are stable in the range of temperature $(<450 \mathrm{~K})$. Compared to previously reported hybrid materials, we find that the A7@graphene system has the lowest decomposition temperature $(450 \mathrm{~K})$, such as the dissociation temperature of the polymeric nitrogen chains encapsulated in CNT $(>5000 \mathrm{~K})^{35}$ or BNNT $(\sim 1400 \mathrm{~K}) .{ }^{43}$ To understand
Table 1 The amount of charge transfer $(\mathrm{e} / \mathrm{N})$ between polymeric nitrogen chains (sheets) and hosting materials and the dissociation temperature of these hybrid systems

\begin{tabular}{lll}
\hline Hybrid materials & $\begin{array}{l}\text { Charge transfer } \\
\text { by Bader analysis method }\end{array}$ & $\begin{array}{l}\text { The dissociation } \\
\text { temperature }\end{array}$ \\
\hline $\mathrm{N}_{8}$ @CNT & 0.05 & $>5000 \mathrm{~K}$ \\
$\mathrm{~N}_{8}$ @graphene & 0.05 & $>5000 \mathrm{~K}$ \\
$\mathrm{~N}_{8}$ @BNNT & 0.04 & $\sim 1400 \mathrm{~K}$ \\
A7@graphene & 0.0049 & $>450 \mathrm{~K}$
\end{tabular}

this difference, we compare A7@graphene with $\mathrm{N}_{8} @ \mathrm{CNT},{ }^{34}$ $\mathrm{N}_{8} @$ @graphene, ${ }^{35} \mathrm{~N}_{8} @$ BNNT $^{43}$ systems systematically. The results are clearly shown in Table 1 . From the results, we know that the charge transfer amount in the A7@graphene system $(0.0049 \mathrm{e} / \mathrm{N})$ is smaller than that in $\mathrm{N}_{8} @ \mathrm{CNT}(0.05 \mathrm{e} / \mathrm{N}), \mathrm{N}_{8}-$ (agraphene $(0.05 \mathrm{e} / \mathrm{N})$ and $\mathrm{N}_{8} @ B N N T(0.04 \mathrm{e} / \mathrm{N})$. As we know that more charge transfer will lead to a strong electrostatic interaction between the polymeric nitrogen chains and hosting materials, which in turn increases the dissociation temperatures of these hybrid materials, which can be the reason why the A7@graphene has the lowest decomposition temperature compared with other hybrid materials. The smaller charge transfer amount in the A7@graphene system should cause a weaker electron interaction between the polymeric nitrogen sheets and graphene sheets, which is just good for the stabilization of the A7@graphene system at ambient conditions, and favors energy release at a lower temperature, which can be taken as a remarkable advantage of the A7@graphene system.

\subsection{Energetic properties analysis}

Compared with the previously reported one or two-dimensional hybrid materials, such as the $\mathrm{N}_{8} @ \mathrm{CNNT}, \mathrm{N}_{8} @$ @graphene (the weight ratio of nitrogen are $13.46 \%$, and $20.58 \%$, respectively), ${ }^{34,35}$ the nitrogen content of the A7@graphene system is significantly improved, and the weight ratio of nitrogen is up to $53.84 \%$, which make it potentially interesting for the applications as a high energy density material. Based on the PBE method, ${ }^{47,48}$ our calculations shows that the energy difference between the hybrid material and decomposition products is (a)

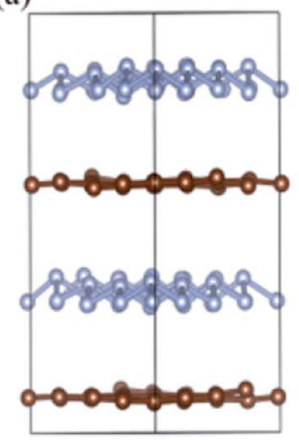

(b)

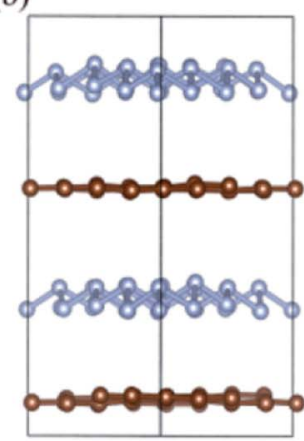

(c)

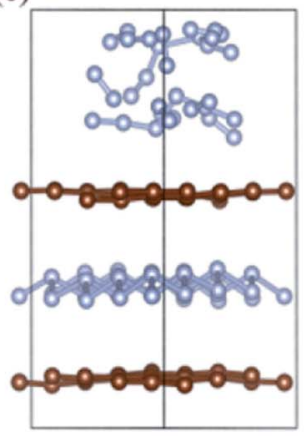

(d)

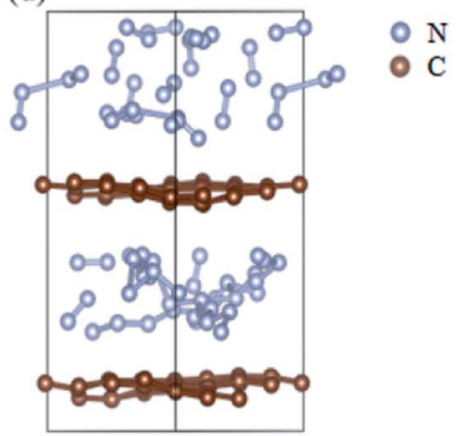

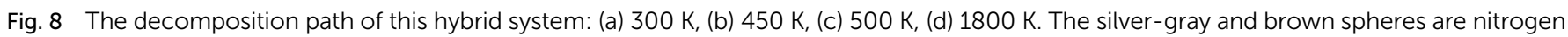
atoms and carbon atoms, respectively. 
Table 2 The calculated values of detonation velocity and detonation pressure of the A7agraphene, and the values of TNT and HMX are reference to the results of Wang et al. ${ }^{58}$

\begin{tabular}{lll}
\hline Compound & $\begin{array}{l}\text { The detonation } \\
\text { velocities }(D)\end{array}$ & $\begin{array}{l}\text { The detonation } \\
\text { pressures }(p)\end{array}$ \\
\hline A7@graphene & 10.94 & 60.95 \\
TNT & 7.37 & 23.46 \\
HMX & 9.11 & 37.32 \\
\end{tabular}

2.78 eV/A7@graphene system (per unit cell) when the A7 polymeric nitrogen is completely decomposed into nitrogen molecules. The corresponding energy density is $5.2 \mathrm{~kJ} \mathrm{~g}^{-1}$. In principle, besides energy density, the detonation velocity and detonation pressure are also important for evaluating the energetic properties of energetic material, so we estimate the detonation velocity and detonation pressure by using the Kamlet-Jacobs simi-empirical equation, ${ }^{58}$ the results are shown in Table 2. Based on the results, we can know that the detonation velocity and detonation pressure of A7@graphene are greatly improved. Thus, the A7@graphene system is a promising candidate for HEDMs.

\section{Conclusions}

In summary, we present a new hybrid structure where polymeric nitrogen sheets are sandwiched between graphene sheets in the form of a three-dimensional crystal. According to theoretical calculations, it is verified that the polymeric nitrogen sheets are stable at ambient pressure and room temperature. Furthermore, the polymeric nitrogen sheets encapsulated in graphene sheets begin to break up into polymeric nitrogen chains or nitrogen gas at above $450 \mathrm{~K}$, and when the temperature is higher $(1800 \mathrm{~K})$, the polymeric nitrogen sheets are completely dissolved into nitrogen gases and release tremendous energy. In addition, the new hybrid material has a higher nitrogen content (the weight ratio of nitrogen is up to $53.84 \%$ ), and the corresponding energy density is $5.2 \mathrm{~kJ} \mathrm{~g}^{-1}$. The hybrid material has a satisfactory energy density, even considerably improved detonation velocity and detonation pressure compared with the traditional powerful energetic materials (TNT, HMX). Based on the electronic properties, it is confirmed that the lower decomposition temperature is ascribed to the lower charge transfer amount between the polymeric nitrogen sheets and graphene sheets which causes a weaker coulomb interaction compared with other hybrid materials, which is just good for the stabilization of the polymeric nitrogen sheets at ambient conditions, and favors the energy release under a gentle way. Thus our study provides a highly promising method for the capture and application of polymeric nitrogen in a controllable manner.

\section{Conflicts of interest}

There are no conflicts to declare.

\section{Acknowledgements}

We acknowledge Zhao Liu, Sen Shao, and Peihao Huang for helpful discussions. This work was financially supported by National Key R\&D Program of China (2018YFA0305900) and the NSFC (116344004, 51320105007, 11604116 and 51602124), Program for Changjiang Scholars and Innovative Research Team in University (IRT1132). We also acknowledge the use of computing facilities at the High Performance Computing Centre of Jilin University.

\section{References}

1 M. M. G. Alemany and J. L. Martins, Phys. Rev. B: Condens. Matter Mater. Phys., 2003, 68, 024110.

2 W. D. Mattson, D. Sanchez-Portal, S. Chiesa and R. M. Martin, Phys. Rev. Lett., 2004, 93, 125501.

3 F. Zahariev, S. V. Dudiy, J. Hooper, F. Zhang and T. K. Woo, Phys. Rev. Lett., 2006, 97, 155503.

4 A. R. Oganov and C. W. Glass, J. Chem. Phys., 2006, 124, 244704.

5 X. L. Wang, Z. He, Y. M. Ma, T. Cui, Z. M. Liu, B. B. Liu, J. F. Li and G. T. Zou, J. Phys.: Condens. Matter, 2007, 19, 425226.

6 K. O. Christe, Propellants, Explos., Pyrotech., 2007, 32, 194204.

7 C. J. Pickard and R. J. Needs, Nat. Mater., 2008, 7, 775-779.

8 X.-Q. Chen, C. L. Fu and R. Podloucky, Phys. Rev. B: Condens. Matter Mater. Phys., 2008, 77, 064103.

9 C. J. Pickard and R. J. Needs, Phys. Rev. Lett., 2009, 102, 125702.

10 Y. Ma, A. R. Oganov, Z. Li, Y. Xie and J. Kotakoski, Phys. Rev. Lett., 2009, 102, 065501.

11 X. Wang, Y. Wang, M. Miao, X. Zhong, J. Lv, T. Cui, J. Li, L. Chen, C. J. Pickard and Y. Ma, Phys. Rev. Lett., 2012, 109, 175502.

12 X. Wang, F. Tian, L. Wang, X. Jin, D. Duan, X. Huang, B. Liu and T. Cui, New J. Phys., 2013, 15, 013010.

13 F. Zahariev, A. Hu, J. Hooper, F. Zhang and T. Woo, Phys. Rev. B: Condens. Matter Mater. Phys., 2005, 72, 214108.

14 J. Zhang, Z. Zeng, H.-Q. Lin and Y.-L. Li, Sci. Rep., 2014, 04358.

15 M. Sun, Y. Yin and Z. Pang, Comput. Mater. Sci., 2015, 98, 399-404.

16 F. Peng, Y. Yao, H. Liu and Y. Ma, J. Phys. Chem. Lett., 2015, 6, 2363-2366.

17 F. Peng, Y. Han, H. Liu and Y. Yao, Sci. Rep., 2015, 16902.

18 F. Peng, Y. Wang, H. Wang, Y. Zhang and Y. Ma, Phys. Rev. B: Condens. Matter Mater. Phys., 2015, 92, 094104.

19 D. Plašienka and R. Martoňák, J. Chem. Phys., 2015, 142, 094505.

20 M. Frost, R. T. Howie, P. Dalladay-Simpson, A. F. Goncharov and E. Gregoryanz, Phys. Rev. B: Condens. Matter Mater. Phys., 2015, 93, 024113.

21 K. Yin, Y. Wang, H. Liu, F. Peng and L. Zhang, J. Mater. Chem. A, 2015, 3, 4188-4194.

22 M. J. Greschner, M. Zhang, A. Majumdar, H. Liu, F. Peng, J. S. Tse and Y. Yao, J. Phys. Chem. A, 2016, 120, 2920-2925. 
23 J. Zhang, A. R. Oganov, X. Li and H. Niu, Phys. Rev. B, 2017, 95, 020103.

24 Z. Liu, D. Li, S. Wei, W. Wang, F. Tian, K. Bao, D. Duan, H. Yu, B. Liu and T. Cui, Inorg. Chem., 2017, 56, 7494-7500.

25 C. Mailhiot, L. H. Yang and A. K. McMahan, Phys. Rev. B: Condens. Matter Mater. Phys., 1992, 46, 14420-14435.

26 J. Uddin, V. Barone and G. E. Scuseria, Mol. Phys., 2006, 104, 745-749.

27 M. I. Eremets, A. G. Gavriliuk, I. A. Trojan, D. A. Dzivenko and R. Boehler, Nat. Mater., 2004, 3, 558-563.

28 X. Wang, K. Bao, F. Tian, X. Meng, C. Chen, B. Dong, D. Li, B. Liu and T. Cui, J. Chem. Phys., 2010, 133, 044512.

29 D. Tomasino, M. Kim, J. Smith and C.-S. Yoo, Phys. Rev. Lett., 2014, 113, 205502.

30 A. E. Gash, T. M. Tillotson, J. H. Satcher, J. F. Poco, L. W. Hrubesh and R. L. Simpson, Chem. Mater., 2001, 13, 999-1007.

31 C. Zhang, C. Sun, B. Hu, C. Yu and M. Lu, Science, 2017, 355, 374-376.

32 W. Zhang, J. Zhang, M. Deng, X. Qi, F. Nie and Q. Zhang, Nat. Commun., 2017, 8, 181.

33 W. Zhang, K. Wang, J. Li, Z. Lin, S. Song, S. Huang, Y. Liu, F. Nie and Q. Zhang, Angew. Chem., Int. Ed., 2018, 57, 2592-2595.

34 H. Abou-Rachid, A. Hu, V. Timoshevskii, Y. Song and L.-S. Lussier, Phys. Rev. Lett., 2008, 100, 196401.

35 V. Timoshevskii, W. Ji, H. Abou-Rachid, L.-S. Lussier and H. Guo, Phys. Rev. B: Condens. Matter Mater. Phys., 2009, 80, 115409.

36 W. Ji, V. Timoshevskii, H. Guo, H. Abou-Rachid and L.-S. Lussier, Appl. Phys. Lett., 2009, 95, 021904.

37 F. Zheng, C. Wang and P. Zhang, J. Comput. Theor. Nanosci., 2012, 9, 1129-1133.

38 I. K. M. Law, L. Liu, A. Xu, K. S. L. Lam, P. M. Vanhoutte, C.-M. Che, P. T. Y. Leung and Y. Wang, Proteomics, 2009, 9, 2444-2456.

39 D. W. Chang, E. K. Lee, E. Y. Park, H. Yu, H.-J. Choi, I.-Y. Jeon, G.-J. Sohn, D. Shin, N. Park, J. H. Oh, L. Dai and J.-B. Baek, J. Am. Chem. Soc., 2013, 135, 8981-8988.

40 W. Xu, T.-S. Lim, H.-K. Seo, S.-Y. Min, H. Cho, M.-H. Park, Y.-H. Kim and T.-W. Lee, Small, 2014, 10, 1999-2005.
41 Y.-F. Lu, S.-T. Lo, J.-C. Lin, W. Zhang, J.-Y. Lu, F.-H. Liu, C.-M. Tseng, Y.-H. Lee, C.-T. Liang and L.-J. Li, ACS Nano, 2013, 7, 6522-6532.

42 Y. Xu, Q. Wang, C. Shen, Q. Lin, P. Wang and M. Lu, A series of energetic metal pentazolate hydrates, Nature, 2017, 549, 78-81.

43 S. Liu, M. Yao, F. Ma, B. Liu, Z. Yao, R. Liu, T. Cui and B. Liu, J. Phys. Chem. C, 2016, 120, 16412-16417.

44 H. Lv, M. yao, Q. Li, R. Liu, B. Liu, Z. Yao, D. Liu, Z. Liu, J. Liu, Z. Chen, B. Zou, T. Cui and B. Liu, Sci. Rep., 2014, 13234.

45 Z. Wu, E. M. Benchafia, Z. Iqbal and X. Wang, Angew. Chem., 2014, 126, 12763-12767.

46 G. Kresse and J. Furthmüller, Phys. Rev. B: Condens. Matter Mater. Phys., 1996, 54, 0163-1829.

47 J. P. Perdew, A. Ruzsinszky, G. I. Csonka, O. A. Vydrov, G. E. Scuseria, L. A. Constantin, X. Zhou and K. Burke, Phys. Rev. Lett., 2008, 100, 136406.

48 A. Vanderlei dos Santos, G. Padilha and M. Monçalves, Solid State Sci., 2012, 14, 269-275.

49 J. H. Monkhorst and D. J. Pack, Phys. Rev. B: Solid State, 1976, 13, 5188-5192.

50 J. Klimeš, D. R. Bowler and A. Michaelides, Phys. Rev. B: Condens. Matter Mater. Phys., 2011, 83, 195131.

51 K. Lee, É. D. Murray, L. Kong, B. I. Lundqvist and D. C. Langreth, Phys. Rev. B: Condens. Matter Mater. Phys., 2012, 82, 081101.

52 X. Wang, F. Tian, L. Wang, T. Cui, B. Liu and G. Zou, J. Chem. Phys., 2010, 132, 024502.

53 S. V. Bondarchuk and B. F. Minaev, Comput. Mater. Sci., 2017, 133, 122-129.

54 Y. Hu, P. Huang, L. Guo, X. Wang and C. Zhang, Phys. Lett. A, 2006, 359, 728-732.

55 M. Ghosh, L. Wang and S. A. Asher, Appl. Spectrosc., 2012, 66, 1013-1021.

56 W. Tang, E. Sanville and G. Henkelman, J. Phys.: Condens. Matter, 2009, 21, 084204.

57 A. Togo, F. Oba and I. Tanaka, Phys. Rev. B: Condens. Matter Mater. Phys., 2008, 78, 134106.

58 G. X. Wang, H. M. Xiao, X. H. Ju and X. D. Gong, Acta Chim. Sin., 2007, 6, 517-524. 